\title{
Hidden Quasars in Ultraluminous Infrared Galaxies
}

\author{
H. D. Tran, M. S. Brotherton, S. A. Stanford, \& W. van Breugel \\ Institute of Geophysics 83 Planetary Physics, Lawrence Livermore \\ National Laboratory, Livermore, CA 94550
}

\begin{abstract}
Many ultraluminous infrared galaxies (ULIRGs) are powered by quasars hidden in the center, but many are also powered by starbursts. A simply diagnostic diagram is proposed that can identify obscured quasars in ULIRGs by their high-ionization emission lines ([O III $] \lambda 5007 / \mathrm{H} \beta \gtrsim 5)$, and "warm" IR color $\left(f_{25} / f_{60} \gtrsim 0.25\right)$.
\end{abstract}

Ultraluminous infrared galaxies (ULIRGs, $L_{I R}>10^{12} L_{\odot}$ ) are an important constituent of our local universe, with luminosities and space densities similar to those of QSOs (Soifer et al. 1987). This led to the suggestion that ULIRGs could contain infant quasars enshrouded in a large amount of dust (Sanders et al. 1988). On the other hand, they may also represent energetic, compact starbursts (Condon et al. 1991). Understanding the dominant energy input mechanism in these ULIRGs - whether it is obscured quasars or intense bursts of star formation - has been the main issue concerning their nature. In order to better understand the energy sources of ULIRGs and the relationship between AGN and starburst activity, we started a spectropolarimetric survey to search for hidden broad emission lines in a sample of ULIRGs that were identified in the cross correlations between the IRAS Faint Source Catalog (FSC) and those of the FIRST (Becker et al. 1995) and Texas (Douglas et al. 1996) radio surveys.

Using the 10-m Keck II telescope, we obtained spectropolarimetric observations of one ULIRG selected from a sample identified in the FIRST-FSC correlation (FF sources, Stanford et al. 1998), and two ULIRGs from the TexasFSC correlation (TF sources, Dey \& van Breugel 1994). The infrared properties of these galaxies are listed in Table 1 . Our results show that only the

Table 1. Infrared Properties

\begin{tabular}{lcccccccc} 
Object & $z$ & $m$ & $\log \left(L_{I R} / L_{\odot}\right)$ & $f_{12}$ & $f_{25}$ & $f_{60}$ & $f_{100}$ & $f_{25} / f_{60}$ \\
\hline FF J1614+3234 & 0.710 & 19.1 & 13.2 & $<0.065$ & $<0.055$ & 0.174 & $<0.54$ & $<0.31$ \\
TF J1020+6436 & $\mathbf{0 . 1 5 3}$ & 19.0 & 12.1 & $<0.095$ & $<0.062$ & 0.86 & 1.24 & $<0.072$ \\
TF J1736+1122 & 0.162 & 18.0 & 12.3 & $<0.081$ & 0.196 & 0.484 & $<3.31$ & 0.404
\end{tabular}

high-ionization Seyfert 2 galaxy TF J1736+1122 is highly polarized, displaying a broad-line spectrum visible in polarized light (Fig. 1a). The other two objects, TF J1020+6436 and FF J1614+3234, exhibit spectra dominated by a population of young (A-type) stars similar to those of " $\mathrm{E}+\mathrm{A}$ " galaxies. They are unpolarized, showing no sign of hidden broad-line regions. The presence of young starburst components in all three galaxies indicates that the ULIRG phe- 

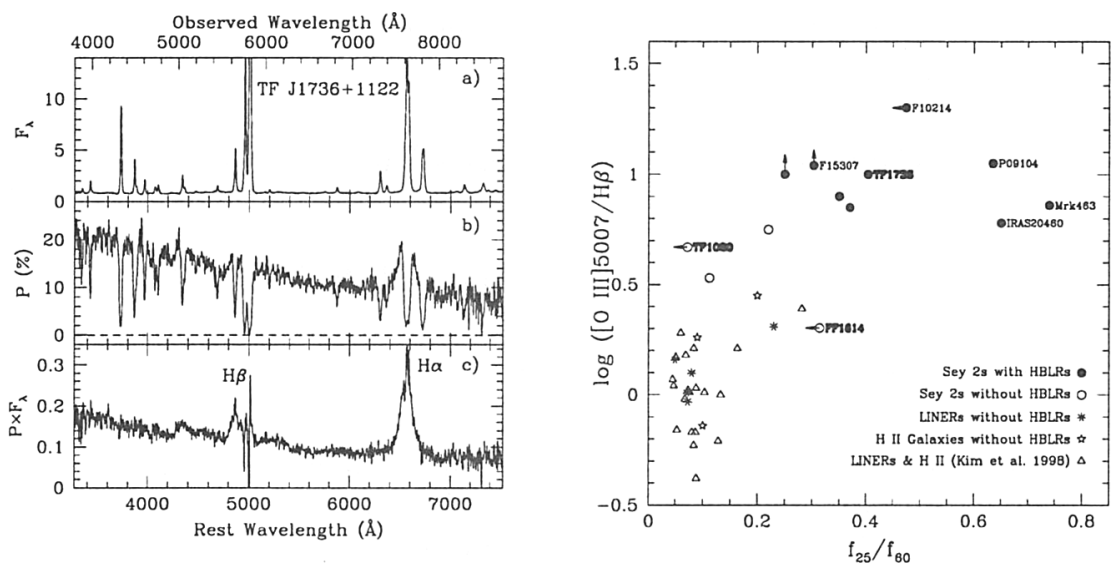

Figure 1. Left panel: Spectropolarimetry of TF J1736+1122. (a) Total flux spectrum, (b) observed degree of polarization, and (c) polarized flux spectrum. Right panel: [O III] $\lambda 5007 / \mathrm{H} \beta$ versus IR color $f_{25} / f_{60}$ for narrow-line ULIRGs in which HBLRs have been searched for. Also plotted in open triangles are all ULIRGs classified as H II and LINERs in Kim et al. (1998). Note the clear tendency for ULIRGs with HBLRs to have warmer IR color and higher excitation spectrum. The opposite holds for LINERs and H II galaxies.

nomenon encompasses both AGN and starburst activity, but the most energetic ULIRGs do not necessarily harbor "buried quasars".

It is of interest to see if it could be determined, from optical spectroscopic and IRAS photometric data alone, whether an ULIRG harbors a genuine quasar or is powered by a starburst. Figure $1 \mathrm{~b}$ shows a plot of the line ratio [O III] $\lambda 5007 / \mathrm{H} \beta$ (narrow), which can serve as an indicator of the ionization level, versus the infrared color $f_{25} / f_{60}$. As can be seen, there is a clear tendency for higher-ionization and warmer Seyfert 2 ULIRGs to show hidden broad-line region (HBLR) indicative of a "buried quasar." Furthermore, Seyfert ULIRGs without HBLRs lie in a region of the diagram similar to that occupied by $\mathrm{H}$ II and LINER galaxies, none of which has been found to have HBLRs.

Acknowledgments. Research at IGPP/LLNL is performed under the auspices of the US Department of Energy under contract W-7405-ENG-48.

\section{References}

Becker, R. H., White, R. L., \& Helfand, D. J. 1995, ApJ, 450, 559

Condon, J. J. et al. 1991, ApJ, 378, 65

Dey, A., \& van Breugel, W. 1994, in Mass-Transfer Induced Activity in Galaxies, ed. I. Shlosman, p. 263

Douglas, J. N. et al. 1996, AJ, 111, 1945

Kim, D.-C., Veilleux, S., \& Sanders, D. B. 1998, ApJ, in press, astro-ph/9806149 
Sanders, D. B. et al. 1988, ApJ, 325, 74

Soifer et al. 1987, ApJ, 320, 238

Stanford, S. A., Stern, D., van Breugel, W., De Breuck, C. 1998, ApJ, in prep. 